University of Nebraska - Lincoln

DigitalCommons@University of Nebraska - Lincoln

Faculty Publications from Nebraska Center for

Materials and Nanoscience, Nebraska Center Materials and Nanoscience

for (NCMN)

2019

Comparative study of topological Hall effect and skyrmions in

NiMnIn and NiMnGa

Wenyong Zhang

Balamurugan Balasubramanian

Ahsan Ullah

Rabindra Pahari

Xingzhong Li

See next page for additional authors

Follow this and additional works at: https://digitalcommons.unl.edu/cmrafacpub

Part of the Atomic, Molecular and Optical Physics Commons, Condensed Matter Physics Commons, Engineering Physics Commons, and the Other Physics Commons

This Article is brought to you for free and open access by the Materials and Nanoscience, Nebraska Center for (NCMN) at DigitalCommons@University of Nebraska - Lincoln. It has been accepted for inclusion in Faculty Publications from Nebraska Center for Materials and Nanoscience by an authorized administrator of DigitalCommons@University of Nebraska - Lincoln. 


\section{Authors}

Wenyong Zhang, Balamurugan Balasubramanian, Ahsan Ullah, Rabindra Pahari, Xingzhong Li, Lanping Yue, Shah R. Valloppilly, Andrei Sokolov, Ralph Skomski, and David J. Sellmyer 


\title{
Comparative study of topological Hall effect and skyrmions in NiMnIn and NiMnGa
}

\author{
Cite as: Appl. Phys. Lett. 115, 172404 (2019); doi: 10.1063/1.5120406 \\ Submitted: 17 July 2019 - Accepted: 9 October 2019 . \\ Published Online: 22 October 2019
}

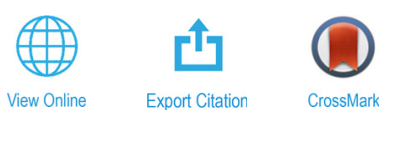

Wenyong Zhang, ${ }^{1,2, a)}$ Balamurugan Balasubramanian, ${ }^{1,2}$ (D) Ahsan Ullah, ${ }^{1,2}$ Rabindra Pahari, ${ }^{1,2}$ (D) Xingzhong Li, Lanping Yue, (D) Shah R. Valloppilly, ${ }^{\top}$ Andrei Sokolov, ${ }^{\top}$ Ralph Skomski, ${ }^{1,2}$ and David J. Sellmyer ${ }^{1,2}$

\author{
AFFILIATIONS \\ ${ }^{7}$ Nebraska Center for Materials and Nanoscience, University of Nebraska, Lincoln, Nebraska 68588, USA \\ ${ }^{2}$ Department of Physics and Astronomy, University of Nebraska, Lincoln, Nebraska 68588, USA

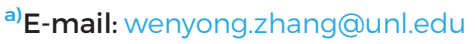

\begin{abstract}
A nonequilibrium rapid-quenching method has been used to fabricate NiMnIn and NiMnGa alloys that are chemically and morphologically similar but crystallographically and physically very different. NiMnGa crystallizes in a $\mathrm{Ni}_{2}$ In-type hexagonal structure, whereas NiMnIn is a cubic Heusler alloy. Both alloys yield a topological Hall effect contribution corresponding to bubble-type skyrmion spin structures, but it occurs in much lower magnetic fields in NiMnIn as compared to NiMnGa. The effect is unrelated to net Dzyaloshinskii-Moriya interactions, which are absent in both alloys due to their inversion-symmetric crystal structures. Based on magnetic-force microscopy, we explain the difference between the two alloys by magnetocrystalline anisotropy and uniaxial and cubic anisotropies yielding full-fledged and reduced topological Hall effects, respectively. Since NiMnIn involves small magnetic fields $(0.02-0.3 \mathrm{kOe})$ at and above room temperature, it is of potential interest in spin electronics.
\end{abstract}

Published under license by AIP Publishing. https://doi.org/10.1063/1.5120406

Skyrmion-containing magnetic materials have recently attracted much attention due to their intriguing physical properties and potential applications in information technology. ${ }^{1-3}$ Skyrmions are defined as solutions of nonlinear field equations characterized by integer topological quantum numbers, ${ }^{4,5}$ which translate into quantized contributions to the anomalous Hall effect known as the topological Hall effect (THE). ${ }^{6,7}$ One challenge is to create and manipulate skyrmions in low magnetic fields and at temperatures above room temperature.

It is well known and widely emphasized in the literature that Dzyaloshinskii-Moriya (DM) interactions facilitate the formation of magnetic skyrmions. ${ }^{3,8-10}$ These interactions require noncentrosymmetric atomic arrangements, realized, for example, in B20 intermetallics such as $\mathrm{MnSi}^{3,8,10}$ in inverse tetragonal Heusler alloys such as $\mathrm{Mn}_{1.4} \mathrm{Pt}_{0.9} \mathrm{Pd}_{0.1} \mathrm{Sn},{ }^{11}$ and in specifically designed thin-film nanostructures with broken inversion symmetry. ${ }^{12,13}$

More generally, there are several definitions of skyrmions, ${ }^{14}$ such as chiral skyrmions and bubble skyrmions. The skyrmions discussed in this paper are of the bubble type. Skyrmions also exist in the absence of DM interactions, which has given rise to the distinction between skyrmions in noncentrosymmetric materials and skyrmions in centrosymmetric materials. ${ }^{6}$ The latter are closely related to magnetic bubbles, which were intensively investigated in the past. ${ }^{15,16}$ However, broken inversion symmetry is not the only crystallographic consideration. Crystallographic chirality and polarity are equally important, and many noncentrosymmetric crystal structures support bubble-type skyrmions but exhibit no net DM interactions. Point-group analysis clarifies the situation and outlines the occurrence of crystal-specific spin structures. ${ }^{17,18}$ For example, some compounds with noncentrosymmetric point groups, such as inverse cubic Heusler and halfHeusler compounds, do not support DM interactions. ${ }^{17}$

Our focus is on a specific aspect of this relationship, namely, on the distinction between cubic and noncubic crystal structures. Centrosymmetric bulk alloys have recently been shown to exhibit skyrmions. ${ }^{2,19,20} \mathrm{NiMnGa}$ is a typical example, which crystallizes in the hexagonal $\mathrm{Ni}_{2}$ In-type structure (centrosymmetric point group $D_{6 \mathrm{~h}}$, space group $\left.\mathrm{P}_{3} / m m c\right)^{2,19}$ and supports bubble-type skyrmions exhibiting quantized THE contribution to the anomalous Hall effect. ${ }^{21}$ The contributions require noncollinear spin structures $S_{1} \cdot\left(S_{2} \times S_{3}\right) \neq 0$ and yield a continuum contribution $\rho_{\mathrm{xy}}$ to the Hall effect that is proportional to $\boldsymbol{S} \cdot(\partial \boldsymbol{S} / \partial x \times \partial \boldsymbol{S} / \partial y)$. For fully developed skyrmions, this THE contribution is quantized.

In this letter, we compare the skyrmionic behavior of cubic NiMnIn produced by using a nonequilibrium process (rapid quenching from the melt) with that of the chemically similar but crystallographically 
very different hexagonal alloy NiMnGa. Both alloys are randomly oriented polycrystalline and have been produced by the same meltspinning method, which is explained elsewhere. ${ }^{22}$ The equiatomic alloys were prepared by arc-melting mixtures of $99.9995 \%$ pure $\mathrm{Mn}$, $\mathrm{Ni}$, and $\mathrm{Ga}$ or In in a pure argon atmosphere and melt spun at a wheel speed of $15 \mathrm{~m} / \mathrm{s}$ to form ribbons of approximate width $3 \mathrm{~mm}$ ( $y$-direction) and thickness $50 \mu \mathrm{m}(z$-direction) and the equiatomic compositions of the NiMnGa and NiMnIn samples were confirmed using energy-dispersive X-ray spectroscopy (EDS).

The crystal structure of the alloys was investigated with a "Rigaku Smart Lab X-ray Diffractometer" using $\mathrm{Cu} \mathrm{K}_{\alpha}$ radiation of a wavelength of $1.5406 \AA$ and a scanning transmission-electron microscope (FEI Tecnai Osiris). A "Quantum Design" physical property measurement system (PPMS) was used to measure the magnetic and electrontransport properties. The magnetic domain structures of the alloys were investigated using "Bruker-ICON" magnetic force microscopy (MFM).

The structural properties of NiMnGa and NiMnIn alloys were investigated using x-ray diffraction (XRD) patterns (Fig. 1) and transmission electron microscopy studies (Fig. 2). Figure 1 shows the XRD patterns of the melt-spun NiMnGa and NiMnIn alloys (black curves) and our Rietveld fittings (red curves). NiMnGa crystallizes in a transition-metal rich derivative of the hexagonal NiAs structure that can be fitted to a $\mathrm{B} 82$ unit cell with the lattice parameters $a=4.15 \AA$ and $c=5.12 \AA$, Fig. 1(a). Prototypes compatible with this fitting include $\mathrm{NaBeSb},{ }^{23}$ which has $\mathrm{Mn}, \mathrm{Ni}$, and $\mathrm{Ga}$ on the $2 \mathrm{a}, 2 \mathrm{~d}$, and $2 \mathrm{c}$ sites, respectively, and $\mathrm{Ni}_{2} \mathrm{In}$, where $\mathrm{Mn}$ and $\mathrm{Ni}$ form a solid solution involving the $2 \mathrm{c}$ and $2 \mathrm{~d}$ sites. No attempts have been made to quantify the degree and nature of the $\mathrm{Mn}-\mathrm{Ni}$ chemical disorder. All alloys mentioned in this paragraph belong to the hexagonal space group $\mathrm{P}_{3} / \mathrm{mmc}$ and therefore to the centrosymmetric point group $\mathrm{D}_{6 \mathrm{~h}}$. The electron diffraction pattern [Fig. 2(b)] obtained from the bright-field TEM image of NiMnGa [Fig. 2(a)] also confirms the NiAs-type hexagonal structure.

The XRD pattern of NiMnIn, Fig. 1(b), can be indexed to a cubic-Heusler unit cell ( $a=6.16 \AA$ ), except two weak-intensity peaks labeled by + , due to the presence of a minority secondary phase. The notoriously low intensity of the Heusler-specific peaks labeled as $*$ in Fig. 1(b) makes it difficult to distinguish between normal cubic Heusler from inverse-cubic and half-Heusler structures. However, the structural model that fits the experimental data with better agreement using Rietveld refinement in Fig. 1(b) was found to be the full Heusler $\mathrm{L} 2_{1}$ structure (prototype $\mathrm{Cu}_{2} \mathrm{MnAl}$, space group $\mathrm{Fm} \overline{3} \mathrm{~m}$, or $\mathrm{O}_{\mathrm{h}}$ ) with

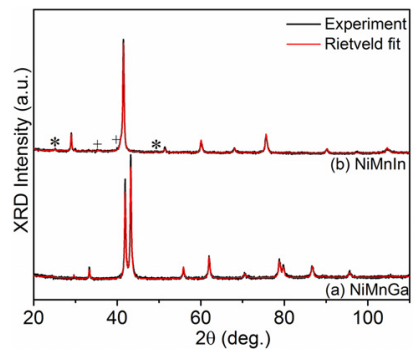

FIG. 1. X-ray diffraction patterns: (a) NiMnGa and (b) NiMnln. The experimental curves (black) are compared with the simulated curves (red) for $\mathrm{Ni}_{2} \ln (\mathrm{a}$ ) and cubic Heusler (b). The x-ray diffraction peaks indicated by the symbols $*$ and + correspond to Heusler-specific reflections and a minority secondary phase, respectively.
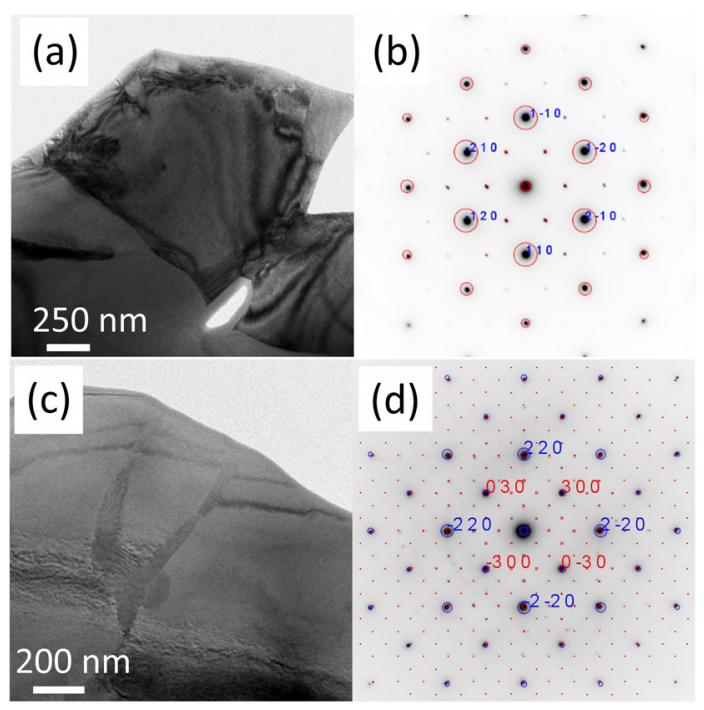

FIG. 2. Structures of (a) and (b) NiMnGa and (c) and (d) NiMnln: (a) and (c) TEM images and (b) and (d) experimental and simulated electron diffraction patterns.

some disorder due to site inversion and vacancies. The lattice parameter obtained for the Heusler phase is $a=6.16 \AA$. Note that the equiatomic NiMnIn is a metastable compound, which often produces a minority secondary phase during the rapid quenching process. ${ }^{24}$ The composition, crystal structure, and quantity of the secondary phase strongly depend on the quench rate. In the present Ni-Mn-In sample, the shoulder around $40.8^{\circ}$ in the XRD pattern is the most intense diffraction peak of the minority phase and its peak position is in good agreement with that of the highest-intensity peak of the $\mathrm{Mn}_{3}$ In-type phase. $^{25}$ The minority phase was also included in the Rietveld analysis, and the fits suggested only about $4.9 \mathrm{wt}$. \% of the minority phase with a lattice parameter of $a=9.38 \AA$. In agreement with the XRD result, the bright-field TEM image of NiMnIn [Fig. 2(c)] shows diffraction patterns corresponding to the main NiMnIn phase with $a=6.16 \AA$ and a minority phase with $a=9.38 \AA$ as indexed in blue and red, respectively, in Fig. 2(d).

Figure 3 shows the field and temperature dependence of the magnetization. The $M(T)$ curves of Fig. 3(a), measured in a field of $100 \mathrm{Oe}$, show that the spin structure is ferro- or ferrimagnetic, with Curie
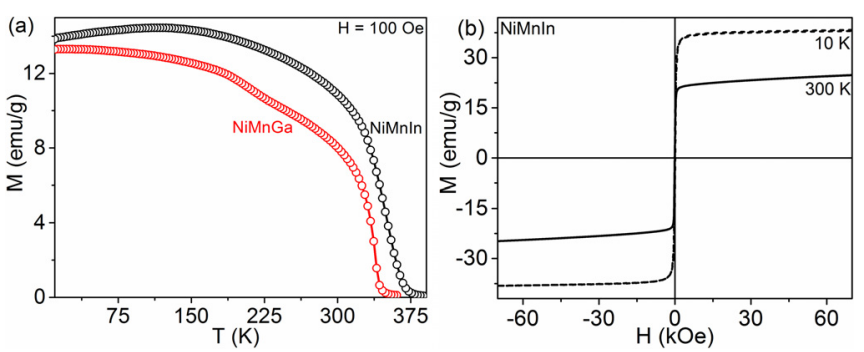

FIG. 3. Temperature and field dependence of the magnetization: (a) thermomagnetic curves of the NiMnGa and NiMnln and (b) field-dependence of NiMnln magnetization measured at $10 \mathrm{~K}$ and $300 \mathrm{~K}$. The field is applied in the z-direction, that is, perpendicular to the ribbons. 
temperatures of $350 \mathrm{~K}$ for NiMnGa and $380 \mathrm{~K}$ for NiMnIn. The saturation magnetization $M_{\mathrm{s}}$ of NiMnIn is $38.0 \mathrm{emu} / \mathrm{g}$ at $10 \mathrm{~K}$ and 24.8 at $\mathrm{emu} / \mathrm{g}$ at $300 \mathrm{~K}$, Fig. 3(b), and the respective magnetizations of $\mathrm{NiMnGa}$ are $80 \mathrm{emu} / \mathrm{g}$ and $51 \mathrm{emu} / \mathrm{g}$ (not shown here). Note that the $\mathrm{Mn}_{3}$ In-type phase is reported to be nonmagnetic at room temperature and exhibit ferrimagnetic properties with $T_{\mathrm{c}} \sim 80 \mathrm{~K}$ with a very small low-temperature magnetization, two orders of magnitude smaller than that of the main NiMnIn phase. ${ }^{23}$ There is also no indication of this secondary phase in the $M(H)$ and $M(T)$ curves of the NiMnIn sample (Fig. 3). Therefore, the minority phase is not expected to influence the high-temperature magnetic properties of the main NiMnIn phase.

There are several ways to experimentally investigate skyrmions. Here, we use Hall-effect analysis ${ }^{2,20,26-30}$ and magnetic-force microscopy (MFM). The link between the two methods is provided by the local spin direction $S(\boldsymbol{r}) \sim \boldsymbol{M}(\boldsymbol{r})$, which determines the MFM contrast and creates an emergent magnetic field $B_{z}$ proportional to the skyrmion density,

$$
\Phi^{\mathrm{z}}=\frac{1}{8 \pi M_{\mathrm{s}}^{3}} \boldsymbol{M} \cdot\left(\frac{\partial \boldsymbol{M}}{\partial x} \times \frac{\partial \boldsymbol{M}}{\partial y}\right) .
$$

The corresponding THE contribution is obtained experimentally by subtracting the known ordinary and anomalous Hall-effect contributions from the total Hall resistivity $\rho_{\mathrm{xy}}$, using

$$
\rho_{\mathrm{xy}}=R_{0} H+R_{\mathrm{s}} M+\rho_{\mathrm{THE}} .
$$

Here, $R_{0}$ is the ordinary Hall coefficient and $R_{\mathrm{s}}$ is the very weakly fielddependent anomalous Hall coefficient. ${ }^{26,28,30}$ Figures $4(\mathrm{a})$ and $4(\mathrm{~b})$ show the measured $\rho_{\text {xy }}$ at different temperatures for NiMnGa and NiMnIn, respectively (circles connected by solid lines). Both samples show very small magnetoresistance $(0.2 \%-0.6 \%$ at $2 \mathrm{~T})$, and therefore, $R_{\mathrm{s}}$ is very weakly field-dependent and simply defined as $R_{\mathrm{s}}=S_{\mathrm{H}} \rho_{\mathrm{xx}}^{2}$ as considered in previous studies. ${ }^{2,26,30} S_{\mathrm{H}}$ is independent of the
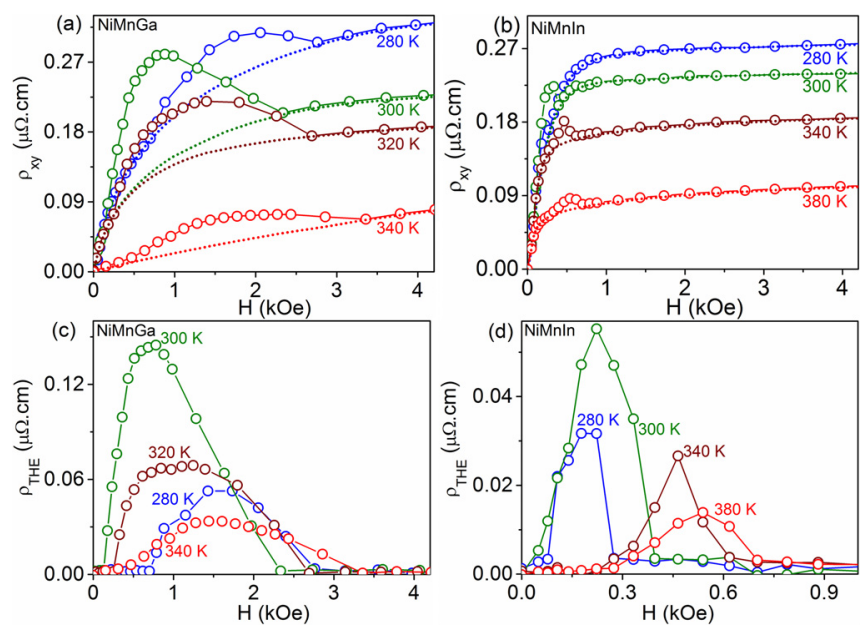

FIG. 4. Hall resistivity $\left(\rho_{\mathrm{xy}}\right)$ measured at various temperatures (circles connected by the solid lines): (a) NiMnGa and (b) NiMnln, where the dotted lines represent the corresponding Hall resistivities without the $\rho_{\text {THE }}$ term extracted from the magnetic measurements using standard Hall effect analysis. Topological Hall resistivities $\left(\rho_{\text {THE }}\right)$ at different temperatures: (c) NiMnGa and (d) NiMnln. magnetic field and $\rho_{\mathrm{xx}}{ }^{2}$ is approximately constant. Equation (2) can be written as

$$
\rho_{\mathrm{xy}}=R_{0} H+S_{H} \rho_{x x}^{2} M+\rho_{\mathrm{THE}} .
$$

When the applied field is larger than $10 \mathrm{kOe}, \rho_{\mathrm{THE}}$ is 0 and $R_{0}$ and $S_{\mathrm{H}} \rho_{\mathrm{xx}}{ }^{2}$ were determined by fitting $\rho_{\mathrm{xy}} / H$ vs $M / H$ in the high field region $(H>10 \mathrm{kOe})$. Since $H$ and $M$ are known (supplementary material, Fig. S1), $\rho_{\mathrm{xy}}$ without the $\rho_{\mathrm{THE}}$ term, simply labeled as $\rho_{\text {xy }}{ }^{\text {extracted }}=R_{0} H+S_{H} \rho_{x x}{ }^{2} M$, can be obtained for the whole field range and are shown as dotted lines in Figs. 4(a) and 4(b). The difference between $\rho_{\mathrm{xy}}$ and $\rho_{\mathrm{xy}}$ extracted yields the corresponding $\rho_{\mathrm{THE}}$ values, which are shown in Figs. 4(c) and 4(d) for NiMnGa and NiMnIn, respectively.

Both alloys exhibit the characteristic THE anomalies (bumps), but in a different way. The NiMnGa bumps have a high intensity but require relatively high fields, whereas the NiMnIn bumps are less intense but occur in low fields, starting at about $0.02 \mathrm{kOe}$ and fully developed in less than $0.3 \mathrm{kOe}$ at room temperature. Bumps of the type shown in Figs. 4(c) and 4(d) are indicative of the topological Hall effect, which is caused by the Berry phase acquired by conduction electrons traveling through skyrmionic spin structures. ${ }^{6,7}$ Note that $M(\mathrm{H})$ curves, Fig. S1, do not show anomalies, and this is probably due to the spin structures of the bulk magnets. ${ }^{2,20}$

To interpret our Hall-effect measurements, we have used magnetic-force microscopy. Figures 5(a) $-5(\mathrm{c})$ and 5(d)-5(f) show the MFM images for NiMnGa and NiMnIn measured in different magnetic fields. The NiMnGa spin structures are consistent with MFM images showing skyrmions in magnetic materials with uniaxial magnetic anisotropy. ${ }^{31-33}$ Each "worm" (band domain) and each spherical domain correspond to one Hall-effect quantum, and the formation of these domains is likely to involve branching from the middle of the relatively thick ribbons. ${ }^{34}$ An excessive applied field leads to the disappearance of skyrmions in NiMnGa, and a nearly uniform magnetization distribution results, Fig. 5(c). Our Lorentz TEM images also show isolated bubble-type skyrmions for the NiMnGa (Fig. S2). For Lorentz TEM measurements, the ribbon samples were made into thin slices having a thickness of about $10-50 \mathrm{~nm}$ using the ion-milling process.

The situation in NiMnIn, Figs. 5(d)-5(f), is very different from that in NiMnGa. The crystal structure of the alloy is cubic, characterized by 6 or 8 easy magnetization directions for each grain, compared

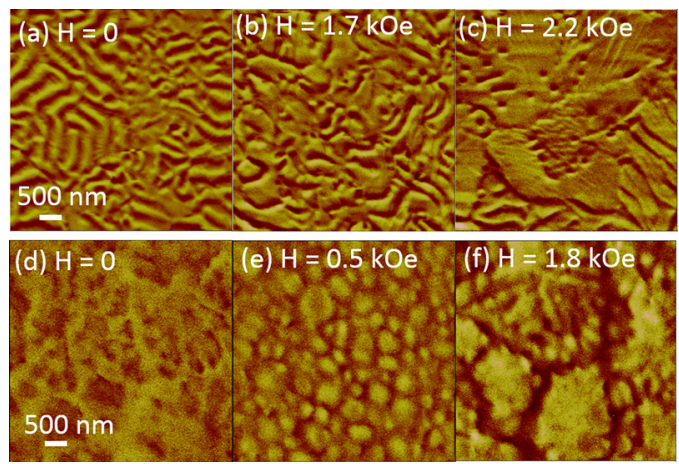

FIG. 5. MFM images measured at various fields for NiMnGa (a)-(c) and NiMnln (d)-(f). 
to 2 directions for uniaxial anisotropy. ${ }^{35}$ Perpendicular magnetization components, corresponding to strong brightness contrast in MFM pictures, cost magnetostatic energy and are therefore avoided whenever possible. Cubic crystals always have easy magnetization directions nearly parallel to the surface. This is seen very clearly in Fig. 5(d), where the MFM contrast is much weaker than in Fig. 5(a). For this reason, the Lorentz-TEM data for thinned cubic alloy sample (not shown in the supplementary material) exhibit virtually no contrast.

When the magnetization is in the $x$ - $y$-plane, as is approximately the case in Fig. $5(\mathrm{~d})$, then Eq. (1) yields $\Phi^{\mathrm{z}}=0$. However, the magnetocrystalline anisotropy of cubic crystals is much lower than that of uniaxial crystals. For example, in the present case, the law of approach to saturation ${ }^{36}$ yields room-temperature anisotropy values of $0.5 \mathrm{Mergs} / \mathrm{cm}^{3}$ for NiMnIn compared to $2 \mathrm{Mergs} / \mathrm{cm}^{3}$ for hexagonal NiMnGa. The low cubic anisotropy means that small magnetic-field changes yield relatively large magnetization changes, especially magnetization components perpendicular to the surface.

It is important to keep in mind that the quantization of the THE originates from the quantization of the integral over the skyrmion density, $\int \Phi^{\mathrm{z}} \mathrm{d} x \mathrm{~d} y$ for thin films or near the bulk surface. ${ }^{6}$ To ensure the quantization, the perpendicular magnetization component must change from $+M_{\mathrm{s}}$ to $-M_{\mathrm{s}}$ (or from $-M_{\mathrm{s}}$ to $M_{\mathrm{s}}$ ) when going from the inside of the skyrmions to the outside. This corresponds to full brightness contrast in MFM pictures.

In conclusion, we have compared the Hall effects in polycrystalline samples with uniaxial (NiMnGa) and cubic (NiMnIn) crystal structures. Both alloys yield a topological Hall-effect contribution despite the absence of Dzyaloshinskii-Moriya interactions. The topological Hall effect is smaller in NiMnIn than in NiMnGa but occurs in a lower magnetic field. We explain this behavior in terms of the cubic anisotropy of NiMnIn, which reduces the topological Hall effect signal. This mechanism, which combines moderate signal strength with high field sensitivity, may be of interest for new spin-electronics applications.

See the supplementary material for the field-dependence of magnetization measured at different temperatures.

This work was primarily supported by the U.S. Department of Energy under the Award No. DE-FG02-04ER46152. It was performed in part in the Nebraska Nanoscale Facility: National Nanotechnology Coordinated Infrastructure and the Nebraska Center for Materials and Nanoscience, which are supported by the National Science Foundation under Award No. NNCI-1542182, and the Nebraska Research Initiative. We thank Lin Zhou and Matthew Kramer from Ames Laboratory for helpful discussions.

\section{REFERENCES}

${ }^{1}$ A. Fert, N. Reyren, and V. Cros, Nat. Rev. Mater. 2, 17031 (2017).

${ }^{2}$ W. H. Wang, Y. Zhang, G. Xu, L. Peng, B. Ding, Y. Wang, Z. Hou, X. Zhang, X. Li, E. Liu, S. Wang, J. Cai, F. Wang, J. Li, F. Hu, G. Wu, B. Shen, and X.-X. Zhang, Adv. Mater. 28, 6887 (2016).

${ }^{3}$ N. Nagaosa and Y. Tokura, Nat. Nanotechnol. 8, 899 (2013).

${ }^{4}$ T. H. R. Skyrme, Proc. R. Soc. London, Ser. A 247, 260 (1958).
${ }^{5}$ C. Naya and P. Sutcliffe, Phys. Rev. Lett. 121, 232002 (2018).

${ }^{6} \mathrm{~S}$. Seki and M. Masahito, Skyrmions in Magnetic Materials (Springer International, Cham, 2016).

${ }^{7}$ D. Xiao, M.-C. Chang, and Q. Niu, Rev. Mod. Phys. 82, 1959 (2010).

${ }^{8}$ P. Bak and H. H. Jensen, J. Phys. C 13, L881 (1980).

${ }^{9}$ N. Kanazawa, S. Seki, and Y. Tokura, Adv. Mater. 29, 1603227 (2017).

${ }^{10}$ T. Yokouchil, S. Hoshino, N. Kanazawa, A. Kikkawa, D. Morikawa, K. Shibata, T. Arima, Y. Taguchi, F. Kagawa, N. Nagaosa, and Y. Tokura, Sci. Adv. 4, eaat1115 (2018).

${ }^{1}$ A. K. Nayak, V. Kumar, T. Ma, P. Werner, E. Pippel, R. Sahoo, F. Damay, U. K. Rößler, C. Felser, and S. S. P. Parkin, Nature 548, 561 (2017).

${ }^{12}$ W. J. Jiang, G. Chen, K. Liu, J. D. Zang, S. G. E. te Velthuis, and A. Hoffmann, Phys. Rep. 704, 1 (2017).

${ }^{13}$ A. Fert, V. Cros, and J. Sampaio, Nat. Nanotechnol. 8, 152 (2013).

${ }^{14}$ K. Everschor-Sitte, J. Masell, R. M. Reeve, and M. Kläui, J. Appl. Phys. 124, 240901 (2018).

${ }^{15}$ P. J. Grundy and S. R. Herd, Phys. Status Solidi A 20, 295 (1973).

${ }^{16}$ C.-W. Chen, Magnetism and Metallurgy of Soft Magnetic Materials (NorthHolland, Amsterdam, 1977).

${ }^{17}$ A. Ullah, B. Balamurugan, W. Zhang, S. Valloppilly, X.-Z. Li, R. Pahari, L.-P. Yue, A. Sokolov, D. J. Sellmyer, and R. Skomski, IEEE Trans. Magn. 55, 7100305 (2019).

${ }^{18}$ M. Kataoka and O. Nakanishi, J. Phys. Soc. Jpn. 50, 3888 (1981).

${ }^{19}$ X. Li, S. Zhang, H. Li, D. A. Venero, J. S. White, R. Cubitt, Q. Huang, J. Chen, L. He, G. van der Laan, W. Wang, T. Hesjedal, and F. Wang, Adv. Mater. 31, 1900264 (2019).

${ }^{20}$ X. Xiao, L. Peng, X. Zhao, Y. Zhang, Y. Dai, J. Guo, M. Tong, J. Li, B. Li, W. Liu, J. Cai, B. Shen, and Z. Zhang, Appl. Phys. Lett. 114, 142404 (2019).

${ }^{21}$ J. C. Loudon, A. C. Twitchett-Harrison, D. Cortés-Ortuño, M. T. Birch, L. A. Turnbull, A. Štefančič, F. Y. Ogrin, E. O. Burgos-Parra, N. Bukin, A. Laurenson, H. Popescu, M. Beg, O. Hovorka, H. Fangohr, P. A. Midgley, G. Balakrishnan, and P. D. Hatton, Adv. Mater. 31, 1806598 (2019).

${ }^{22}$ B. Balamurugan, B. Das, W. Y. Zhang, R. Skomski, and D. J. Sellmyer, J. Phys.: Condens. Mater. 26, 064204 (2014).

${ }^{23}$ C. Tiburtius and H.-U. Schuster, Z. Naturforsch. B 32, 1133 (1977).

${ }^{24}$ X. Z. Li, W. Y. Zhang, S. R. Valloppilly, and D. J. Sellmyer, Sci. Rep. 9, 7762 (2019).

${ }^{25}$ Q. Zhang, D. Li, W. B. Cui, J. Li, and Z. D. Zhang, J. Appl. Phys. 106, 113915 (2009).

${ }^{26}$ S. X. Huang and C. L. Chien, Phys. Rev. Lett. 108, 267201 (2012).

${ }^{27}$ K. G. Rana, O. Meshcheriakova, J. Kübler, B. Ernst, J. Kare, R. Hillebrand, E. Pippel, P. Werner, A. K. Nayak, C. Felser, and S. S. P. Parkin, New J. Phys. 18, 085007 (2016).

${ }^{28}$ M. Leroux, M. J. Stolt, S. Jin, D. V. Pete, C. Reichhardt, and B. Maiorov, Sci. Rep. 8, 15510 (2018).

${ }^{29}$ E. V. Vidal, H. Schneider, and G. Jakob, Phys. Rev. B 83, 174410 (2011).

${ }^{30}$ A. Hrabec, J. Sampaio, M. Belmeguenai, I. Gross, R. Weil, S. M. Chérif, A. Stashkevich, V. Jacques, A. Thiaville, and S. Rohart, Nat. Commun. 8, 15765 (2017).

${ }^{31}$ J. C. Gallagher, K. Y. Meng, J. T. Brangham, H. L. Wang, B. D. Esser, D. W. McComb, and F. Y. Yang, Phys. Rev. Lett. 118, 027201 (2017).

${ }^{32}$ A. Soumyanarayanan, M. Raju, A. L. Oyarce, A. K. C. Tan, M.-Y. Im, A. P. Petrović, P. Ho, K. H. Khoo, M. Tran, C. K. Gan, F. Ernult, and C. Panagopoulos, Nat. Mater. 16, 898 (2017).

${ }^{33}$ J. Brandão, D. A. Dugato, R. L. Seeger, J. C. Denardin, T. J. A. Mori, and J. C. Cezar, Sci. Rep. 9, 4144 (2019).

${ }^{34}$ A. Hubert and R. Schäfer, Magnetic Domains (Springer-Verlag, Berlin, 1998).

${ }^{35}$ R. Skomski, Simple Models of Magnetism (University Press, Oxford, 2008).

${ }^{36} \mathrm{H}$. Kronmüller and M. Fähnle, Micromagnetism and the Microstructure of Ferromagnetic Solids (University Press, Cambridge, 2003), pp. 176-177. 